\title{
ON THE GRÜSS INEQUALITY FOR UNITAL 2-POSITIVE LINEAR MAPS
}

\author{
SRIRAM BALASUBRAMANIAN
}

Abstract. In a recent work, Moslehian and Rajić have shown that the Grüss inequality holds for unital $n$-positive linear maps $\phi: \mathscr{A} \rightarrow B(H)$, where $\mathscr{A}$ is a unital $C^{*}$-algebra and $H$ is a Hilbert space, if $n \geqslant 3$. They also demonstrate that the inequality fails to hold, in general, if $n=1$ and question whether the inequality holds if $n=2$. In this article, we provide an affirmative answer to this question.

Mathematics subject classification (2010): Primary: 46L05, 47A63; Secondary: 47B65. Keywords and phrases: Grüss inequality, $C^{*}$-algebra, $n$-positive, completely positive.

\section{REFERENCES}

[1] T. ANDo, Topics on Operator Inequalities, Division of Appl. Math., Research Institute of Applied Electricity, Hokkaido Univ., Sapporo, 1978.

[2] B. Blackadar, Operator Algebras: Theory of $C^{*}$-Algebras and von Neumann Algebras, Encyclopaedia of Mathematical Sciences, Vol. 122, 2006, ISBN 978-3-540-28517-5.

[3] M. D. Chоi, Some Assorted Inequalities for Positive linear maps on $C^{*}$-algebras, J. Oper. Th., 4 (1980), 271-285.

[4] G. GRÜss, Über das Maximum des absoluten Betrages von $\frac{1}{b-a} \int_{a}^{b} f(x) g(x) d x-\frac{1}{(b-a)^{2}} \int_{a}^{b} f(x) d x$ $\int_{a}^{b} g(x) d x$, Math. Z. 39 (1934), 215-226.

[5] M. S. Moslehian, R. Rajić, A Grüss inequality for n-positive linear maps, Linear Algebra Appl. 433 (2010), 1555-1560.

[6] V. Paulsen, Completely Bounded Maps and Operator Algebras, Cambridge University Press, 1st edition, 2003.

[7] I. PERIĆ, R. RAJIĆ, Grüss inequality for completely bounded maps, Linear Algebra Appl. 390 (2004), 287-292.

[8] P. F. RENAUd, A matrix formulation of Grüss inequality, Linear Algebra Appl. 335 (2001), 95-100.

[9] J. G. StAmpfLI, The norm of a derivation, Pacific J. Math. 33 (1970), 737-747.

[10] T. TAKASAKI, J. Tomiyama, On the geometry of positive maps in matrix algebras, Math. Z. 184 (1983), 101-108. 